
A. MOORE*
M. $\mathrm{KOCH}$
K. MUELLER
M. STUKE

\section{Precise laser ablation processing of black widow spider silk}

Max-Planck-Institut f. biophys. Chemie, Laser Chemical Processing 011, P.O. Box 2841, 37077 Goettingen, Germany

\section{Received: 31 March 2003/Accepted: 4 April 2003 \\ Published online: 5 June 2003 • (C) Springer-Verlag 2003}

ABSTRACT Contact-free precise laser processing of black widow spider (Latrodectus hesperus) dragline silk by laser ablation at $\lambda=157 \mathrm{~nm}$ is achieved. At this wavelength, the small optical penetration depth, below $100 \mathrm{~nm}$, allows efficient and gentle material removal above the ablation threshold of $\Phi_{\mathrm{th}}=29 \mathrm{~mJ} / \mathrm{cm}^{2}$. The ablation rate in $\mathrm{nm} /$ pulse is measured against laser fluence $\Phi$ and simultaneously calibrated with ultrapure polymethylmethacrylate (PMMA). Ripple formation in fiber ablation can be overcome by a suitable combination of ablation removal and laser polishing steps using exposure sequences at different irradiation angles $\alpha$, such as vertical at $0^{\circ}$ followed by oblique irradiation at $70^{\circ}$. In this way ripples are destroyed until the fiber is automatically smoothed over the entire laser-exposed length. This allows precise laser ablation processing of spider silk fibers and other highly absorbing materials without affecting the bulk mechanical, and other material, properties.

PACS 61.41.+e; 61.46.+w; 61.80.Ba; 61.82.Pv; 62.25.+g
1

\section{Introduction}

The dragline silk of spiders is the toughest polymer known. It is fabricated by the spider at ambient temperatures without waste or toxic by-products [1]. How does the spider achieve this? Can the material be reproduced synthetically [2]? By now, much of the protein sequence [3] for spider silk is known and can be generated [4]; yet, the protein thus produced is only a sticky liquid. The key to the spider silk's superior material properties lies in the protein folding [5] and long range order [6] that is permitted by the protein sequence. To date, attempts to create the silk fibers from genetically engineered precursors have not fully reproduced the superior properties of the natural fiber. The best genetically engineered silk fiber [7] so far is equally as tough as the natural fiber with only one third the strength of silk produced by the spider, but it can be expected, that future synthesis improvements will be successful in fully reproducing the outstanding properties of spider silk. Recently much has been discovered about how strength is attained from molecular folding and longrange order within natural spider silk fibers [8]. Nanoscale control of material properties [9-11] is key for major breakthroughs [12] and advances [13] in applications using optical [14] properties including lasing [15]. Precise contact-free $[16,17]$ processing [18] of spider silk is highly attractive, in particular, if its outstanding properties [19] describe the use of high absorption vacuum ultraviolet (VUV) laser ablation processing at $157 \mathrm{~nm}$ both in vacuo and can be maintained. In the following, we

Fax: +49-551/201-1330, E-mail: mstuke@gwdg.de

* Permanent address: Dept. of Biological Sciences, Univ. of the Pacific, Stockton, CA 95211 (USA) Supplementary material to this paper is available in electronic form on Springer Verlag's server at http://dx.doi.org/10.1007/s00339-003-02193-6 when exposed to an inert 700 mbar nitrogen $\left(\mathrm{N}_{2}\right)$ gas. High values of light absorption in organic polymers can be achieved by short wavelength [20] or/and short [21,22] pulse irradiation. If the sample is exposed to a laser fluence (pulse energy per unit area) above a threshold value $\Phi_{\text {th }}$, highly efficient and fast removal of material can be achieved without damage to the remaining bulk.

\section{$2 \quad$ Laser ablation of spider silk}

The ablation behavior of spider silk and the resulting surface morphology are shown in Fig. 1a,b. Single strands of black widow (Latrodectus hesperus) major ampullate (MA) silk were placed on blocks of polymethylmethacrylate (PMMA) so that both materials were simultaneously irradiated vertically at $0^{\circ}$ angle of incidence. U1trapure samples of the well characterized PMMA [23] were used to calibrate the laser fluence $\Phi$. In a chamber that was either empty ( $<10$ mbar) or filled with 700 mbar $_{2}$, all samples were exposed to a $157 \mathrm{~nm}$ VUV excimer laser (Lambda Physik LPF 205) with a pulse duration of $18 \mathrm{~ns}$ and a repetition rate of up to $5 \mathrm{~Hz}$. At this wavelength, the light penetration depth into the fiber sample is only about $100 \mathrm{~nm}$ allowing precise processing. The fluence was varied by changing the energy of the laser or, for higher fluences, by focusing the beam with spherical or cylindrical $\mathrm{CaF}_{2}$ lenses. The depth of material removed by ablation was measured by surface profilometry (Dektak) and Scanning Electron Microscopy, SEM (Phillips XL20). To exclude modification by the electron beam during SEM, samples were imaged at low accelerat- 
ing voltage $(3 \mathrm{KV})$ after being sputter coated with $40 \mathrm{~nm}$ of gold.

The amount of spider silk ablated depends on laser fluence and the number of pulses. Figure 1a shows the amount of material removed per pulse as a function of the laser fluence.

There is a characteristic threshold fluence, $\Phi_{\mathrm{th}}=29 \mathrm{~mJ} / \mathrm{cm}^{2}$, below which no material is removed from the silk.
Above threshold fluence the amount of ablation per pulse increases with fluence. Figure $1 \mathrm{~b}$ shows spider silk fibers that have been exposed to various numbers of pulses at a fluence of $50 \mathrm{~mJ} / \mathrm{cm}^{2}$.
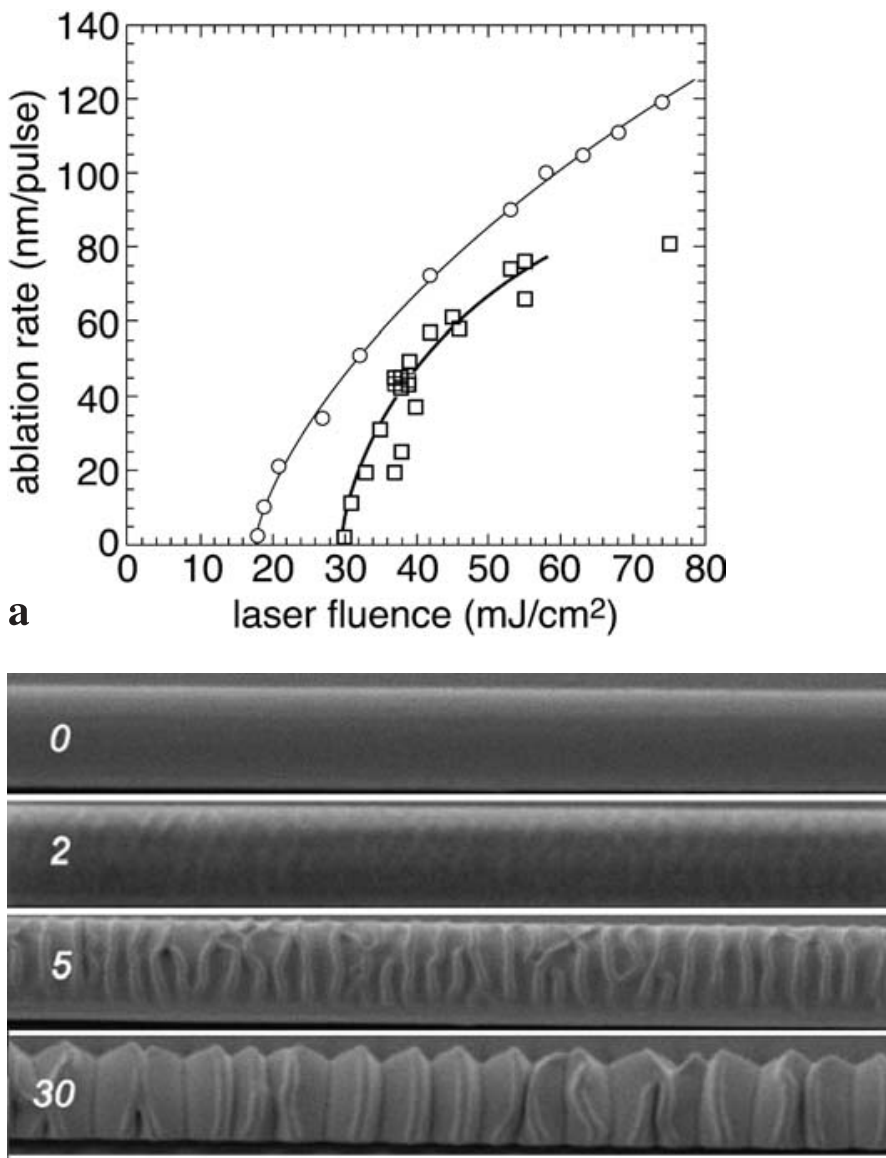

b

a

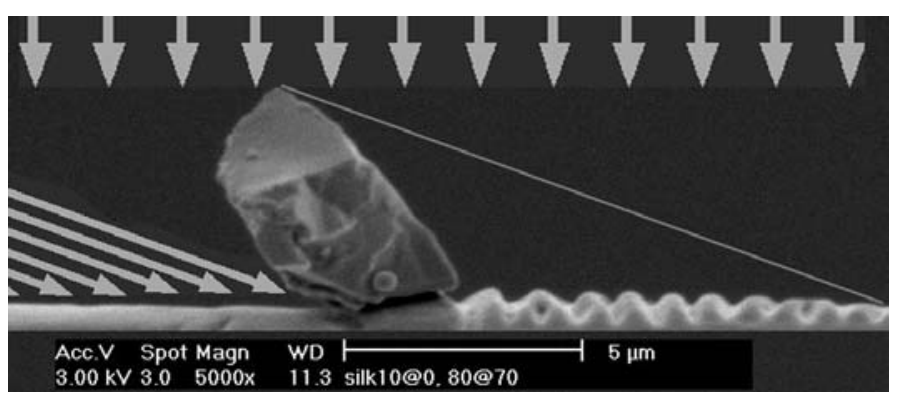

b
FIGURE 1 a Ablation behavior of black widow major ampullate spider silk. Ablation rate in $\mathrm{nm} /$ pulse versus laser fluence for spider silk (bottom, squares) and PMMA (top, circles). Polymethylmethacrylate (PMMA) was irradiated simultaneously with the silk and used as a reference to calibrate the fluence. b Ripples on the ablated surface of spider silk fibers (diameter $3 \mu \mathrm{m}$ ) for different numbers $n=0,2,5,30$ of vertical $\left(\alpha=0^{\circ}\right)$ laser shot exposure. For a given fluence (here $50 \mathrm{~mJ} / \mathrm{cm}^{2}$ ), both amplitude (peak-to-trough depth) and periodicity (peak-to-peak distance along the fiber axis) increase with the number of pulses
FIGURE 2 a A smooth, ablated surface is obtained by a two-step process. The effective laser fluence $\Phi_{\text {eff }}$ experienced by any laser exposed surface section depends on the irradiation angle $\alpha$ such that $\Phi_{\text {eff }}=\Phi_{\mathrm{o}} \cos \alpha$, where $\Phi_{\mathrm{o}}$ is the fluence within the laser beam. Thus, silk surfaces that are parallel to the fiber axis will not be ablated in an $\alpha=70^{\circ}$ treatment because the fluence they experience is only $34 \%$ of $\Phi_{\mathrm{o}}$ and therefore clearly below threshold, whereas the more perpendicular surfaces of the ripples experience a higher fluence above threshold. Consequently, they are ablated until smooth. b Laser ablation polished spider fiber. The fiber was first laser irradiated vertically (incident angle $\alpha=0^{\circ}$ ) with 10 pulses at $157 \mathrm{~nm}$ and a fluence of $36 \mathrm{~mJ} / \mathrm{cm}^{2}$. Next, the fiber was irradiated with the same light but at an incident angle $\alpha=70^{\circ}$. The oblique laser treatment came from the left side. The arrows on the image indicate the directions of the two laser treatments. Ripples that formed during the first treatment are still present to the right of a dust grain, where the surface was shaded from the $70^{\circ}$ treatment. The surface to the left of the dust grain, which experienced both treatments, is as smooth as the untreated surface but $500 \mathrm{~nm}$ lower. The original surface can be seen directly underneath the dust grain, which was not exposed to laser light 
As ablation occurs, ripples form on the surface of the silk fiber. These ripples increase in amplitude (peak-totrough distance) and in "wavelength" (peak-to-peak distance along the fiber axis) as the number of pulses increases. This ripple formation has been observed on other fibers, including silkworm silk [24] and synthetic polymers [25]. Schollmeyer [26] and colleagues have found these ripples on every organic fiber tested and propose that they form as internal stress is released during the ablation process.

With this modified surface morphology, measurement of the material properties is not straightforward: the presence of ripples makes standard methods of tensile testing invalid because a serrated edge will cause stress concentrations at the troughs of the ripples. The new geometry severely weakens the thread, whether material properties have been changed or not. This induced weakness limits the mechanical uses of the fiber. Consequently, we searched for and found a method to automatically smooth the fiber by destroying and gently removing these ripples.

\section{3}

\section{Laser polishing of ablated fibers}

To create a smooth surface, we use a two-step process (see Fig. 2). First, we irradiate the silk vertically at $\alpha=0^{\circ}$ angle of incidence (perpendicular to the fiber axis) in order to remove material. This also creates ripples. Then we irradiate the silk obliquely (nearly parallel to the fiber axis) to ablate the ripples away. In the oblique beam, with an angle of incidence $\alpha$, surfaces that are parallel to the fiber axis experience a much lower effective fluence $\Phi_{\text {eff }}=$ $\Phi_{0} \cos \alpha$ than do surfaces that are nearly perpendicular to the fiber axis and are exposed to $\Phi_{0}$ (see Fig. 2a).

Thus, the fluence experienced by the smoothed surface is below threshold so that new ripples cannot form. The change in surface structure with each treatment is neatly illustrated in Fig. 2b.

First, we irradiate the fiber vertically $\left(\alpha=0^{\circ}\right)$ with 10 pulses at a fluence of $\Phi_{\mathrm{o}}=36 \mathrm{~mJ} / \mathrm{cm}^{2}$. Then we irradiate it with 80 pulses at the same fluence $\Phi_{\mathrm{o}}$ but at an angle of incidence of $\alpha=70^{\circ}$. The actual fluence experienced by each surface is $\Phi_{\text {eff }}=\Phi_{\mathrm{o}} \cos \alpha$. On the fiber happens to be a grain of dust, which masks different sections on the surface from the two treatments. Directly underneath the dust is a pedestal of silk that was shaded from both the $0^{\circ}$ and the $70^{\circ}$ treatments and, therefore, shows the original surface. Silk immediately to the right of the dust grain was exposed only to the $\alpha=0^{\circ}$ treatment because it was shaded from the $\alpha=70^{\circ}$ treatment. Here, one sees the ripples that formed during the $\alpha=0^{\circ}$ treatment. To the left of the dust grain the fiber was exposed to both treatments. Here the surface is as smooth as the original surface, but lower by $500 \mathrm{~nm}$. The smooth, ablated surface is at the same depth as the troughs of the ripples.

\section{$4 \quad$ Material properties}

Dragline silk was collected on two-pronged forks. A single strand of MA silk was separated from the rest of the dragline. From a $45 \mathrm{~mm}$ length section of spider silk, two samples were taken. One was exposed to the laser treatment and then tensile tested. The
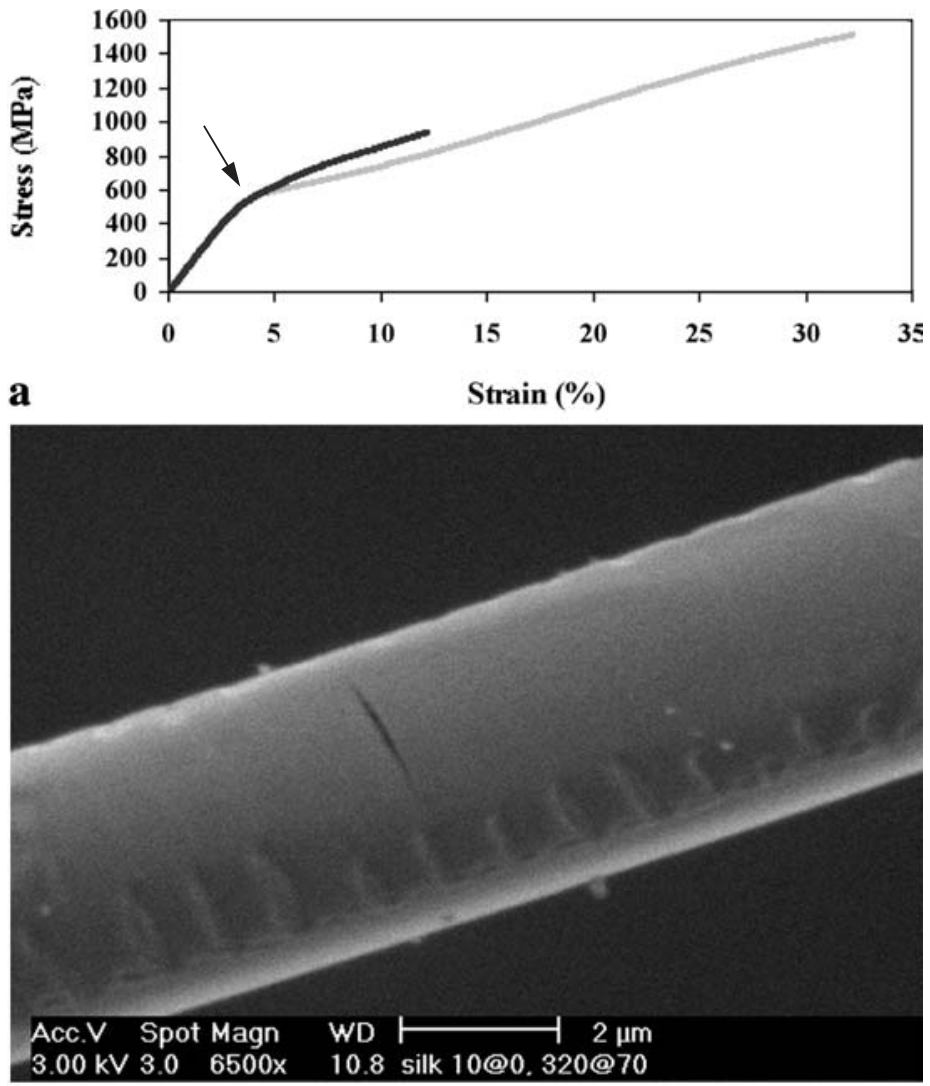

b
FIGURE 3 a Tensile material properties. Stress-strain curves measured for a laser ablated spider silk sample (black) and its adjacent control strand (grey). As can be seen, the yield point (arrow) and the stiffness (slope of the curve) before and after yield are identical. The paths of the two curves are statistically indistinguishable up to the breaking point of the ablated silk. The earlier breaking point halves the strength (breaking stress) and reduces the extensibility (strain at breaking) of the fiber. Only the properties associated with the breaking point differ significantly between the two treatments. The reason for the earlier breaking point is shown in Fig. 3b. b Ablated fiber after tensile testing. Cracks, such as the one shown here, form on the ablated surface as the silk is stretched. Ultimately, they cause the fiber to break earlier than the natural silk. These cracks were always associated with the small indentations left behind by the ripples at the edge of the ablation field. Thus, the indentations occur only on fibers that were laser exposed on one side. Fibers that are laser processed on all sides (see Fig. 4) do not have these indentations 
other was tensile tested without laser treatment as a control because the material properties of spider silk can vary with spinning conditions and between spiders $[1,27]$. Each test fiber was exposed on one side to 10 pulses of $157 \mathrm{~nm}$ laser light at an incident angle of $\alpha=0^{\circ}$ followed by 320 pulses of the same laser at an incident angle of $\alpha=70^{\circ}$.

Figure 3 a shows stress-strain curves for one ablated sample and its adjacent control.

Table 1 lists average values for all eighteen measured samples.

Results from the untreated silk are identical to those previously reported [28]. As can be seen, the shape of the curve, the yield point (point of inflection on the curve), and the pre-yield and post-yield stiffnesses (slopes of the curve) are identical between ablated and untreated silks. These similarities confirm that the bulk of the silk material is not affected by the laser treatment. The only way in which the laser ablation affects material properties is that it caused the silk to break earlier along the same stress-strain curve. This difference, however, causes a two-fold difference in strength and extensibility.

Although the ablated silk is still stronger than wool and other biomaterials [29], it is desirable to maintain the high strength of the natural spider silk. SEM images of the tensile-tested samples reveal that the early breaking point is due to cracks (see Fig. 3b) generated upon stretching.

These cracks are always associated with small indentations left behind by

\begin{tabular}{lcc}
\hline Property & Untreated & Ablated \\
\hline Pre-Yield Stiffness (GPa) & $14 \pm 2$ & $13 \pm 2$ \\
Post-Yield Stiffness (GPa) & $3.6 \pm 1$ & $3.8 \pm 1$ \\
Yield Stress (MPa) & $340 \pm 90$ & $300 \pm 90$ \\
Yield Strain (\%) & $2.7 \pm 0.4$ & $2.6 \pm 0.4$ \\
Breaking Point: & & \\
Strength (MPa) & $1200 \pm 300$ & $630 \pm 180$ \\
Extension (\%) & $29 \pm 7$ & $13 \pm 4$ \\
\hline
\end{tabular}

TABLE 1 Material properties. Mean and standard deviation are presented for material properties of ablated silk compared to untreated silk. Note that only the breaking point properties differ significantly between the two samples

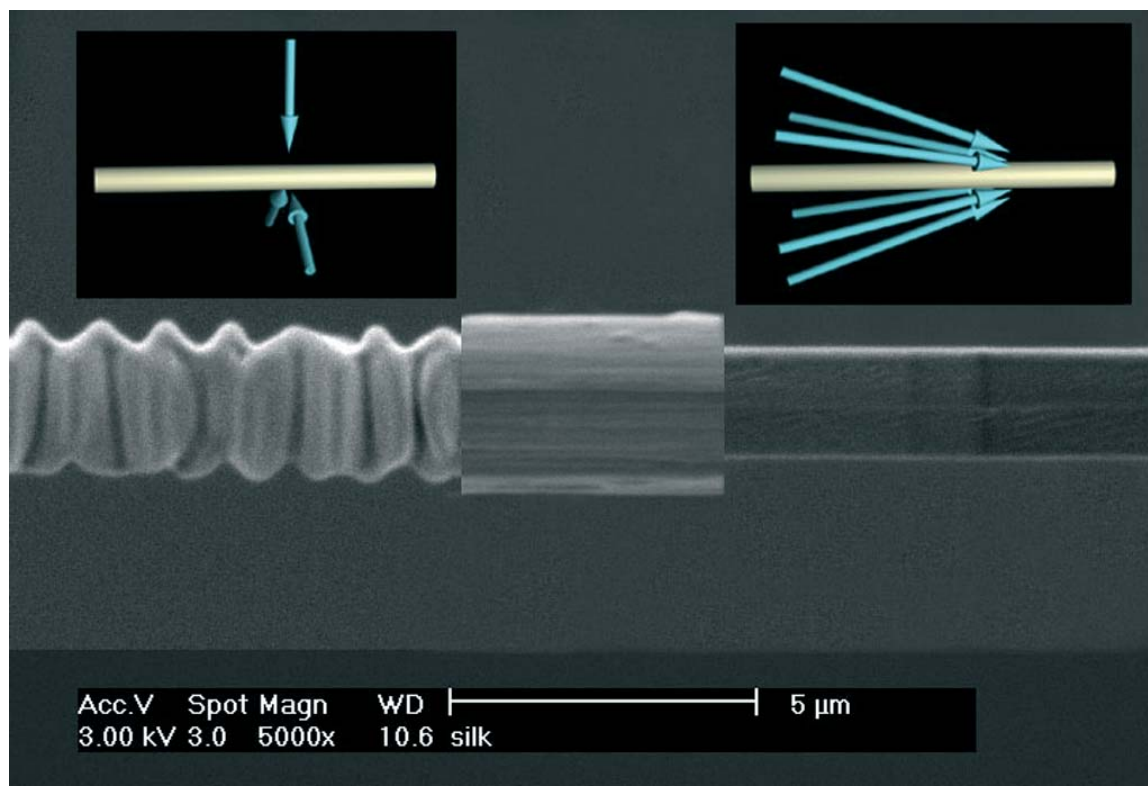

FIGURE 4 Smoothly ablated silk. This is a composite of three scanning electron micrographs showing the laser polishing ablation process. The middle image displays an untreated black widow spider MA dragline silk. To the left is an image of a silk fiber after a 3-fold vertical $\left(\alpha=0^{\circ}\right)$ exposure of the whole fiber with 10 pulses each at $157 \mathrm{~nm}\left(\Phi=36 \mathrm{~mJ} / \mathrm{cm}^{2}\right)$ from 3 angles, see inserted schematic above this section of the fiber. In the projection to the fiber's cross-sectional axis, the angular increments between the 3 removal exposures are $3 \times 120^{\circ}$ keeping $\alpha=0^{\circ}$. The resulting fiber has ripples on all three sides and a roughly triangular cross-section. The image to the right is a silk fiber after the 6-fold polishing step, irradiated - in addition - at $\alpha=70^{\circ}$ from 6 angles with 250 pulses each, see insert above that fiber section. The angular increments between the 6 polishing steps were $6 \times 60^{\circ}$ maintaining $\alpha=70^{\circ}$ in each case. This high angle laser ablation polishing removes ripples, no matter where they occur, until the fiber is completely smooth over the entire laser exposed length the ripples at the edge of the laserexposed ablation field. Because of the circular cross-section of the spider silk fiber, this edge experiences a higher incident angle than the rest of the ablated surface. Therefore, it was occasionally not polished smooth. We have solved this problem by ablating the fiber homogeneously from all sides.

5

\section{Homogeneously smooth ablated fibers}

To avoid edge effects, we expose all sides of a spider silk fiber first to the vertical $\left(\alpha=0^{\circ}\right)$ removal irradiation, then to the oblique $\left(\alpha=70^{\circ}\right)$ polishing irradiation. Since all sides of the fiber are ablated, there are no edges to the ablation field and, therefore, polishing is complete. Figure 4 shows the result of this process.

We vertically irradiate single strands of black widow MA dragline silk homogeneously with 11 pulses at $157 \mathrm{~nm}$ at three angles (see insert top left in Fig. 4). This creates ripples on all sides. We then laser polished the ripples away using 250 pulses at $\alpha=70^{\circ}$ from 6 evenly spaced directions (see insert top right in Fig. 4). Doubling the increment number of polishing angles from three to six compared to the removal process assures that the edges of the ablation fields are polished until smooth. The whole process produces a completely smooth silk fiber without indentations along the entire laser exposed silk fiber sample.

\section{6}

\section{Conclusions}

Ripple formation in laser ablation of silk fibers can be overcome by a suitable combination of removal and laser polishing steps using an appropriate exposure sequence at different angles. In this way ripples are automatically destroyed until the fiber is smooth over the entire laser exposed length. This allows precise laser ablation processing of spider silk fibers and other highly absorbing materials without affecting the mechanical and other properties of the bulk material.

ACKNOWLEDGEMENTS This work was in part financially supported by grants BMBF 13N5398/7 (M.S.), DFG STU 108/6-3 (M.S.) and \#01-12165, National Science Foundation, (A.M.) 


\section{REFERENCES}

1 F. Vollrath, D.P. Knight: Nature 410, 541 (2001)

2 D. Kaplan et al. (eds.): Silk Polymers: Materials Science and Biotechnology (American Chemical Society, Washington D.C. 1993)

3 P.A. Guerette, D.G. Ginzinger, B.H.F. Weber, J.M. Gosline: Science 272, 112 (1996)

4 C.Y. Hayashi, R.V. Lewis: Bioessay 23, 750 (2001) and references therein

5 P.A. Fields, Y.S. Kim, J.F. Carpenter, G.N. Somero: J. Exp. Biol. 205, 1293 (2002) and references therein

6 A.H. Simmons, C.A. Michal, L.W. Jelinski: Science 271, 84 (1996)

7 A. Lazaris, S. Arcidiacono, Y. Huang, J.F. Zhou, F. Duguay, N. Chretien, E.A. Welsh, J.W. Soares, C.N. Karatzas: Science 295, 472 (2002)

8 C. Viney: In Structural Biological Materials: Design and Structure-Property Relationships, ed. by M. Elices (Pergamon,
New York 2000) pp. 293-331, and references therein.

9 D.H. Lowndes, D.B. Geohegan, A.A. Puretzky, D.P. Norton, C.M. Rouleau: Science 273 898 (1996)

10 R.H. Baughman, A.A. Zakhidov, W.A. de Heer: Science 297, 787 (2002) and references therein

11 T. Jaworek, D. Neher, G. Wegner: Science 279, 57 (1998)

12 H. Sirringhaus, H. Tessler, R.H. Friend: Science 280, 1741 (1998)

13 E. Yablonovitch: Science 289, 557 (2000)

14 C. Hettich, C. Schmitt, J. Zitzmann, S. Kuhn, I. Gerhardt, V. Sandoghdar: Science 298, 385 (2002)

15 M.S. Gudiksen: Nature 415, 617 (2002) and references therein

16 O. Lehmann, M. Stuke: Science 270, 1644 (1995) and references therein

17 M.C. Wanke, O. Lehmann, K. Müller, Q. Wen, M. Stuke: Science 275, 1284 (1997)

18 R. Schuster, V. Kirchner, P. Allongue, G. Ertl: Science 289, 98 (2000)
19 J. Gatesy, C. Hayashi, D. Motriuk, J. Woods, R. Lewis: Science 291, 2603 (2001)

20 D. Basting, U. Stamm: Z. Phys. Chem. 215, 1575 (2001) and references therein

21 S. Kueper, M. Stuke: "Femtosecond UV Excimer Laser Ablation" Appl. Phys. B 44, 199 (1987)

22 S. Kueper, M. Stuke: Appl. Phys. Lett. 54, 4 (1989)

23 M. Lapczyna, M. Stuke: Appl. Phys. A 66, 473 (1998) and references therein.

24 D. Knittel, K. Schafer, E. Schollmeyer: Ang. Makromol. Chem. 172, 79 (1989)

25 S. Tokino, Y. Wada, M. Ueda, T. Wakida: En'I Gakkaishi 54, 520 (1998)

26 W. Kesting, Th. Bahners, E. Schollmeyer: Appl. Surface Sci. 46, 326 (1990)

27 F. Vollrath, B. Madsen, Z. Shao: Proc. R. Soc. Lond. B 268, 2339 (2001)

28 N.L. Vuong, A.M.F. Moore: Am. Zool. 38, 165A (1998)

29 J. Vincent: Structural Biomaterials (Princeton University Press, 1990) and references therein 